\title{
Observation of enhanced optical spring damping in a macroscopic mechanical resonator and application for parametric instability control in advanced gravitational-wave detectors
}

\author{
S. W. Schediwy, C. Zhao, L. Ju, and D. G. Blair \\ School of Physics, The University of Western Australia, Nedlands, Western Australia 6009, Australia \\ P. Willems \\ California Institute of Technology, Pasadena, California 91125, USA
}

(Received 22 December 2006; published 16 January 2008)

\begin{abstract}
We show that optical spring damping in an optomechanical resonator can be enhanced by injecting a phase delay in the laser frequency-locking servo to rotate the real and imaginary components of the optical spring constant. This enhances damping at the expense of optical rigidity. We demonstrate enhanced parametric damping which reduces the $Q$ factor of a $0.1-\mathrm{kg}$-scale resonator from $1.3 \times 10^{5}$ to $6.5 \times 10^{3}$. By using this technique adequate optical spring damping can be obtained to damp parametric instability predicted for advanced laser interferometer gravitational-wave detectors.
\end{abstract}

DOI: 10.1103/PhysRevA.77.013813

PACS number(s): 42.65.Yj, 04.80.Nn, 95.55.Ym

The LIGO laser interferometer gravitational-wave $(\mathrm{GW})$ detectors have achieved design sensitivity $\sim<3$ $\times 10^{-23} / \sqrt{\mathrm{Hz}}$ around $100 \mathrm{~Hz}$ and a sensitivity range for neutron star binary inspiral events of $\sim 15 \mathrm{Mpc}$. Other detectors are in operation, commissioning or planning [1]. Even with this high sensitivity, the expected rate of GW observation is less than one event per year. For this reason, advanced detectors are planned for which the amplitude sensitivity will be increased by a factor of 10 , thereby increasing the search volume by three orders of magnitude. To achieve this sensitivity, the laser power in the optical arm cavities needs to be increased towards $1 \mathrm{MW}$.

At such power levels it has been shown that optical spring effects will be strong. In particular, there is a risk of a three mode parametric instability (PI) [2], which can cause uncontrolled mechanical oscillation of the arm cavity test masses in the frequency range from 30 to $120 \mathrm{kHz}$ [3]. Unfortunately, the conditions used to achieve high detector sensitivity are concurrent with the conditions which favor PI.

GW detectors require a PI gain $R_{\mathrm{PI}}<1$ for stability. The magnitude of $R_{\mathrm{PI}}$ is directly proportional to the mechanical quality factor $Q_{m}$ of the test-mass mechanical modes [2]. Passive damping using ring dampers in contact with the test mass can be used to reduce $R_{\mathrm{PI}}$ below unity, but only at the expense of some thermal noise degradation [4]. Alternatively, optical spring damping applied by means of an additional cavity can in principle be noise free [5]. This form of damping is based on a two-mode optomechanical interaction. It utilizes the intrinsic phase lag in a high finesse cavity to apply a radiation pressure damping force to the cavity mirrors. Optical spring damping has been widely observed in radio-frequency and microwave cavities [6] and relatively recently in both microscopic [7] and macroscopic optical cavities $[8,9]$.

There are several problems with optical damping in the context of laser interferometers. First, to obtain significant damping it is necessary that the phase delay, set by the storage time, should be about $\pi / 2$. To obtain sufficient storage time requires either very long or very high finesse cavities. In addition, since the storage time is fixed, the phase delay is frequency dependent so the damping constant cannot be optimized for all potentially unstable modes.

In this paper, we demonstrate that the optical spring damping term can be enhanced at the expense of the optical stiffness term by utilizing a cavity frequency control system to supply the optimal phase shift. We show that the damping agrees with theory and demonstrate a strong reduction of the quality factor $Q_{m}$ in a macroscopic resonator coupled to a $10-\mathrm{cm}$ optical cavity. Finally, we extrapolate our results to the design of a practical damping cavity for an advanced GW detector. We show that by using servo loop enhancement adequate optical spring damping can be obtained.

PI is predicted to arise in high-power laser interferometers due to a three-quantum process whereby optical carrier photons $\omega_{0}$ resonantly scatter from test-mass mechanical mode phonons $\omega_{m}$ to create high-order mode photons at a frequency $\omega_{h}$ which satisfies the energy conservation equation $\omega_{0}=\omega_{h}+\omega_{m}[2,10]$. For high-optical-power interferometers such as Advanced LIGO, the parametric gain $R_{\mathrm{PI}}$ is expected to be in the range of 10-100. Thermal tuning of the test-mass radius of curvature has been proposed as means to alter the mechanical-mode frequencies of the test-mass and therefore break the energy conservation condition. However, the high density of mechanical and optical modes makes it difficult to avoid at least several situations with $R \sim 10-100$. On the other hand, a reduction of $Q_{m}$ by a factor $\sim 100$ is sufficient to create unilateral stability. Clearly, if the mechanical $Q$ factors of most of the test-mass mechanical modes (typically $Q_{m} \sim 10^{7}-10^{8}$ ) could be significantly reduced, $R_{\mathrm{PI}}$ could be reduced below unity, eliminating the instability problem.

Optical springs arise due to the length dependence of the radiation pressure force, which has a phase lag stemming from the cavity storage time. The real part of the complex optical spring constant $k_{o p t}$ changes the cavity stiffness, which in turn affects the mechanical-mode frequency $\omega_{m}$ $=2 \pi f_{m}$. The imaginary component gives rise to parametric damping. The addition of an electronic phase delay $\theta$ in the laser frequency servo system allows the real and imaginary components of the optical spring constant to be rotated by an angle $\theta$ in the complex plane. It is therefore possible to maxi- 


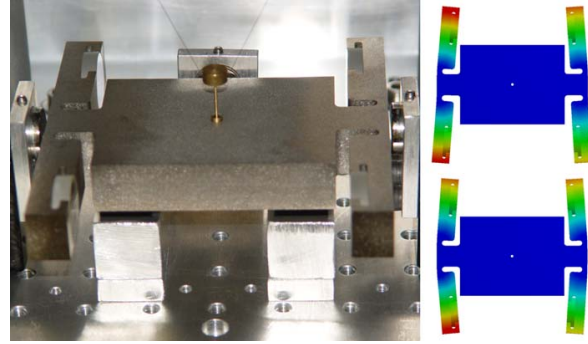

FIG. 1. (Color online) Photograph of the suspended mechanical resonator showing the supporting brass pin, steel wire, and the three magnets used for eddy current damping of the low-frequency $(<10 \mathrm{~Hz})$ torsional and tilting modes (left) and the mechanical mode shape of the resonator's two lowest modes; $f_{m 1}=722 \mathrm{~Hz}$ (top) and $f_{m 2}=748 \mathrm{~Hz}$.

mize damping at the expense of elastic stiffness. The enhanced complex spring constant $k_{e n}$ is derived using servo control theory and is given by

$$
k_{\text {en }}=k_{\text {opt }} /\left(1+G_{0} e^{i \theta}\right),
$$

where $G_{0}$ and $\theta$ and are magnitude and phase of the servo open loop gain and $k_{\text {opt }}$ is given by [5]

$$
k_{o p t}=\frac{16 \pi P_{i n}}{c \lambda} \frac{\sqrt{T}}{(1-R)^{5 / 2}} \frac{f_{o s} / f_{p}}{\left[1+\left(f_{o s} / f_{p}\right)^{2}\right]^{2}}\left(1+\frac{2 i\left(f_{m} / f_{p}\right)}{1+\left(f_{o s} / f_{p}\right)^{2}}\right) .
$$

Here $P_{\text {in }}$ is the input power, $c$ is the speed of light, $\lambda$ is the wavelength of the laser light, $T$ is the mirror power transmissivity, $R$ is the mirror power reflectivity, $f_{o s}$ is the cavity offset frequency, $f_{p}$ is the cavity pole frequency, and $f_{m}$ is the mechanical-mode frequency. For each cavity mirror, the loss $L$, transmissivity $T$, and reflectivity $R$ must naturally sum to unity. That is, $1=T+R+L$. Note from Eq. (1) that the servo gain $G_{0}$ acts to suppress the optical spring while the phase factor $\theta$ can be tuned to enhance it.

To test parametric cold damping, we chose to use an optomechanical resonator with mass $\omega_{m}$ and $Q_{m}$ about $1 / 100$ th of a typical advanced detector test mass. We chose a cavity length of $l=10 \mathrm{~cm}$ and finesse of $F=4500$, which are typical of parameters that might be chosen to tranquilize an advanced detector test mass. The cavity storage time is too low for significant parametric damping without additional phase delay.

The resonator, shown in Fig. 1, consists of a niobium $\mathrm{H}$-shaped section with attached mirrors between the arms. The resonator was machined from a single piece of niobium, annealed to minimize the mechanical loss, and etched to remove surface defects. Two normal modes $f_{m 1}=722 \mathrm{~Hz}$ and $f_{m 2}=748 \mathrm{~Hz}$ (Fig. 1) are strongly coupled to the optical cavity. Four Newport supermirrors were bonded to the resonator using low-mechanical-loss yacca gum bonding [11], thereby forming a pair of $10-\mathrm{cm}$ optical cavities (optical properties given in Table I). Only one cavity was used for this experiment. The resonator was suspended using a loop of steel wire attached to a tapered brass pin inserted into a matching tapered hole at the resonator's center. It was placed in a
TABLE I. Measured optical cavity parameters.

\begin{tabular}{lc}
\hline \hline Parameters & Value and uncertainty \\
\hline Length $l$ & $100 \pm 1 \mathrm{~mm}$ \\
Reflectivity $R$ & $0.999312(53)$ \\
Finesse $\mathcal{F}$ & $4560 \pm 35$ \\
Cavity pole $f_{p}$ & $164.5 \pm 1.3 \mathrm{kHz}$ \\
\hline \hline
\end{tabular}

vacuum tank with a pressure of $2.5 \pm 0.1 \times 10^{-4}$ mbar. The $Q$ factor of $f_{m 2}$ was measured to be $1.31 \pm 0.15 \times 10^{5}$. The cantilever mode of the arms has an effective mass of $0.0323(5) \mathrm{kg}$.

A 1064-nm Laser Zentrum Hannover (model No. LZH 300E) Nd:YAG laser was locked to the optical cavity using a Pound-Drever-Hall (PDH) reflected servo loop. The highfrequency path feeds to the laser piezoelectric transducer (PZT) while the slow path controls the laser temperature. No mode cleaner or intensity stabilization was implemented. Though the beam jitter and the laser intensity noise will convert to radiation pressure noise applied to the mechanical resonator, this noise is random in phase as compared to the mechanical resonance and therefore should not contribute to change the mechanical $Q$ factor. Because the excitation of the mechanical modes in the measurement is much larger than the background noise, the beam jitter and intensity noise have little effect on the measurement. Figure 2 shows the layout of the servo loop. Amplifier 1 is a first-order low-pass filter with gain $G_{1}$ and corner frequency $f_{c}$. Closed-loop transfer functions were recorded between the test points "in" and "out" with the excitation signal injected at "ex." The total loop gain $G_{0}$ was set to be $20.3=\mathrm{B} 10.2 \mathrm{~dB}$ at the mechanical resonance frequency. The loop phase $\theta$ was measured for three different filter corner frequencies $f_{c}$ and recorded in Table II.

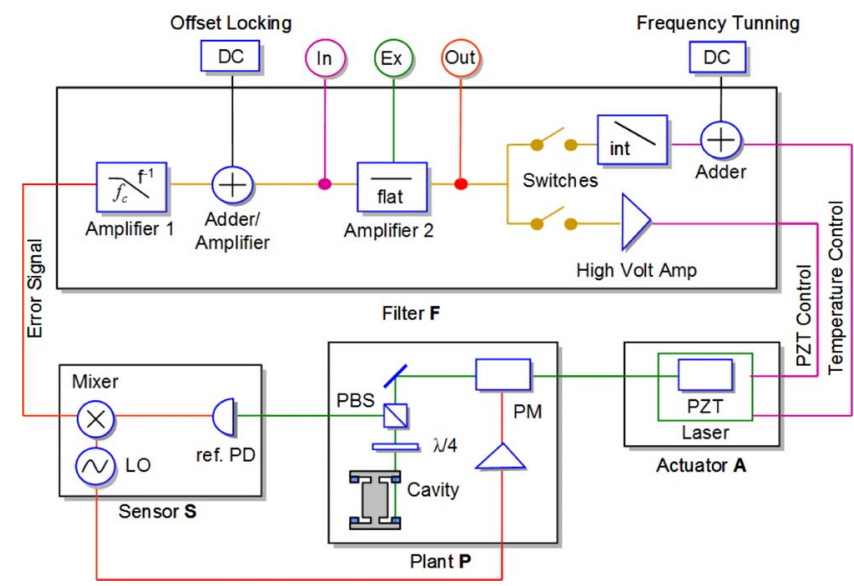

FIG. 2. (Color online) Schematic layout of the servo loop used to lock the laser frequency to the cavity length. A filtered PDH reflected error signal is used to feedback to the laser PZT and temperature. The filter corner frequency $f_{c}$ and gain $G_{1}$ are set at "amplifier 1." LO is the local oscillator, ref. PD is the reflected beam photodiode, PBS is the polarizing beam splitter, $\lambda / 4$ is the quarterwave plate, PM is the phase modulator, and PZT is the laser piezoelectric crystal. 
TABLE II. Phase delay values and uncertainties measured from the closed loop transfer function at the resonator's mechanical frequency $f_{m}$ for three settings of "amplifier 1's" corner frequency $f_{c}$.

\begin{tabular}{lc}
\hline \hline Corner frequency $f_{c}$ & Phase delay $\theta$ \\
\hline $300 \mathrm{~Hz}$ & $-67^{\circ} \pm 2^{\circ}$ \\
$1000 \mathrm{~Hz}$ & $-33^{\circ} \pm 2^{\circ}$ \\
$3000 \mathrm{~Hz}$ & $-12^{\circ} \pm 2^{\circ}$ \\
\hline \hline
\end{tabular}

We measured $Q_{m}$ as a function of cavity offset frequency $\Delta f$ for the $748-\mathrm{Hz}$ mechanical mode, as a function of both laser power and servo loop parameters. Normal modes were acoustically excited through residual coupling to a loudspeaker mounted on the vacuum tank, and the ring-down method was used to measure $Q_{m}$. In the case of instability, no excitation was required and a ring-up curve was used to determine $Q_{m}$. The mechanical ring-down (or ring-up) was measured using the reflected optical error signal down converted to $1 \mathrm{~Hz}$ and fitted to an exponential. Multiple measurements were used to determine uncertainties.

The cavity-locking point was varied by applying a dc offset as shown in Fig. 2. Ten repeat measurements of the $Q$ factor were made at each of the 14-16 different offset frequency points for the three servo loop corner frequencies. Figure 3 shows the Inverse $Q, Q_{m}^{-1}$ for the $748-\mathrm{Hz}$ mode as a function of cavity offset frequency $f_{\text {os }}$. The plot clearly

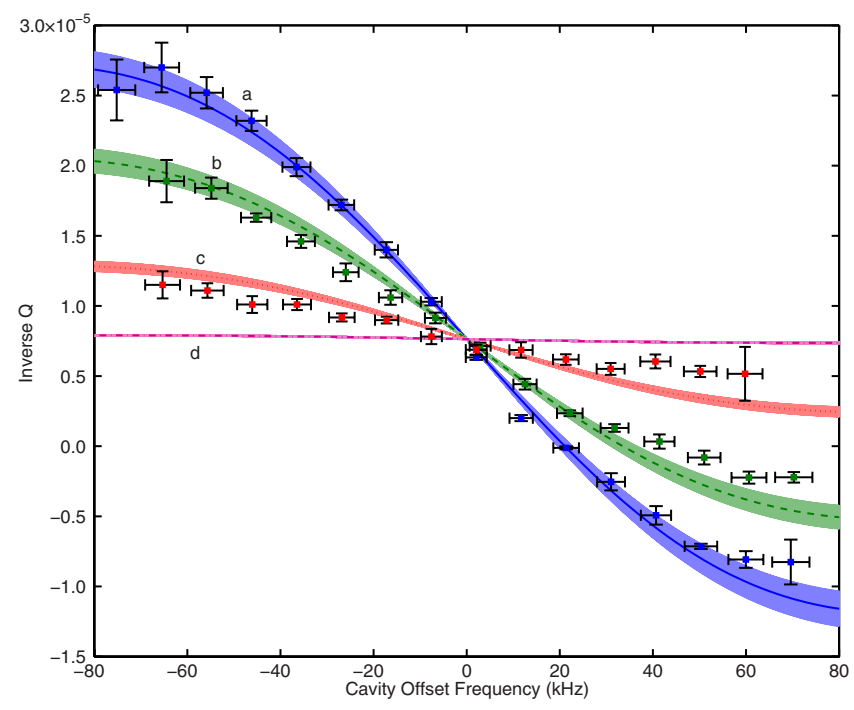

FIG. 3. (Color online) Inverse $Q$ of the $f_{m}=748 \mathrm{~Hz}$ mode as a function of cavity offset frequency for three different configurations of the servo loop corner frequency $f_{c}$, resulting in three different phase delays $\theta$. The stored power is equal for all curves. The measurement data points are shown with one standard deviation error bars. The four curves are the theoretical predictions calculated from the imaginary part of $k_{e n}$. All the input parameters were experimentally determined (there are no free parameters) with the shaded regions represent their uncertainty. Curve $d$ is the expected inverse $Q$ variation if no enhanced optical spring effect were present $\left(\theta=0^{\circ}\right)$. This curve has the same general shape as the other curves, but clearly the magnitude of the $Q$-factor change is much smaller.

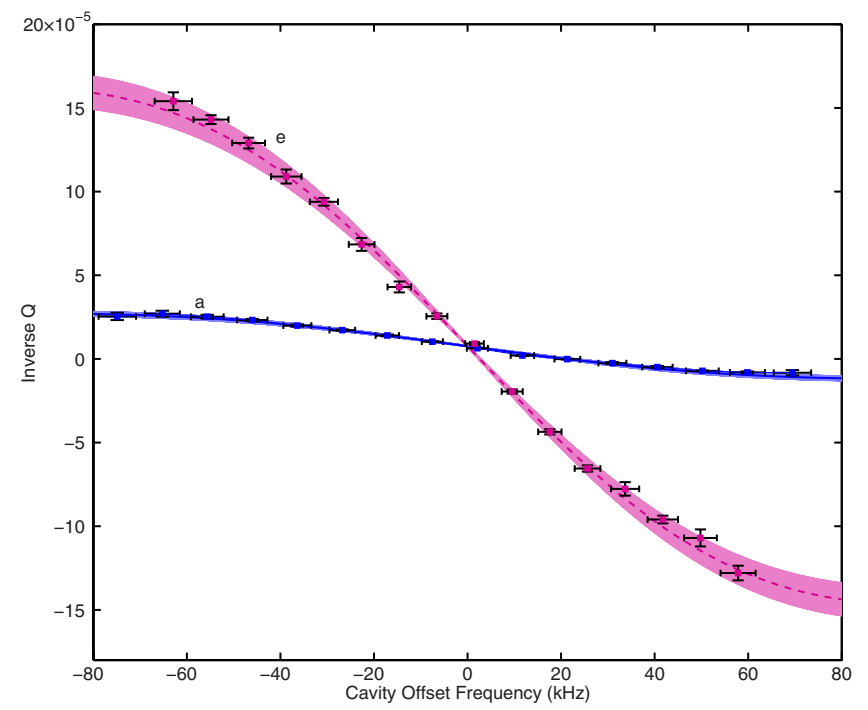

FIG. 4. (Color online) Inverse $Q$ of the $748-\mathrm{Hz}$ mode as a function of offset frequency for two settings of cavity stored power. The phase lag was set at $\theta=-67^{\circ}$ for both measurements. Curve $a$ is the same as curve $a$ from Fig. 3, while curve $e$ was measured using the maximum laser power available for this experiment.

shows enhancement in both $Q_{m}^{-1}$ (with negative cavity offset) and negative losses (with positive cavity offset) as a function of servo phase delay $\theta$. Figure 4 shows $Q_{m}^{-1}$ for the $748-\mathrm{Hz}$ mode as a function of offset frequency $f_{\text {os }}$ for two settings of laser power. Curve (a) is the same as curve (a) in Fig. 3, while curve (e) was obtained using the maximum laser power available for this experiment. The plot shows that at maximum cavity offset lock, the mechanical $Q$ factor was reduced from $1.31 \times 10^{5}$ to $6.51 \times 10^{3}$ with negative cavity offset and parametric instability was produced with a parametric gain $R_{\mathrm{PI}}$ of 18.3 with positive cavity offset. For a detailed description of the experimental design, including the full list of values used to determine $k_{\text {en }}$ from Eq. (2), see Ref. [12].

We now estimate the tranquilizer cavity requirements for PI damping in an advanced interferometer. We assume typical test-mass parameters for an advanced interferometer. In previous work, it has been shown that parametric instabilities excite mechanical modes from about 30 to $120 \mathrm{kHz}$. Typical mechanical modes have an effective mass $\sim 10 \mathrm{~kg}$, and the mechanical mode quality factor needs to be reduced from $\sim 10^{8}$ to a few times $10^{6}$ [3]. For strong damping, it is necessary that the mechanical modes fall within the cavity linewidth. This restricts the product of cavity length $l$ and finesse $\mathcal{F}$.

We compare two cases: (a) $l=10 \mathrm{~cm}, \mathcal{F}=4500$ and (b) $l$ $=1 \mathrm{~cm}, \mathcal{F}=30000$. In both cases, the servo loop has a gain of 2 at $10 \mathrm{kHz}$ with a $-10-\mathrm{dB} /$ decade decrease with increasing mechanical mode frequency $f_{m}$ and a similarly decreasing phase delay resulting in $-80^{\circ}$ at $10 \mathrm{kHz}$ and $-120^{\circ}$ at $100 \mathrm{kHz}$. This produces a stable control loop with maximum enhancement damping at the higher end of the PI frequency range.

Enhanced damping can be used to increase the amount of damping but there is a trade-off; it requires a finite servo 


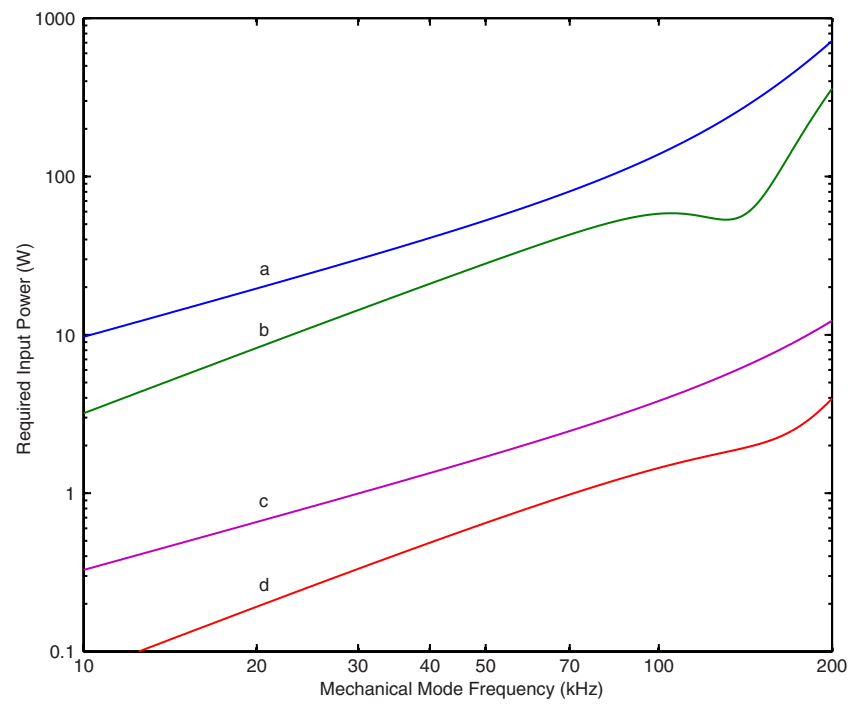

FIG. 5. (Color online) Input power as a function of mechanicalmode frequency required to achieve a mechanical-mode $Q$ factor of $10^{6}$, for a cavity offset frequency of $f_{o s}=-f_{p} / 2$. Curve $a: 10-\mathrm{cm}$ cavity, $F=4500$, without phase enhancement. Curve $b$ : same cavity, with phase enhancement. Curve $c$ : 1 -cm cavity, $F=30000$, without phase enhancement. Curve $d$ : same cavity, with phase enhancement.

gain, which in turn reduces the magnitude of the optical damping, as shown in Eq. (1). Figure 5 shows the input laser power required to damp a test-mass mechanical mode to a $Q$ factor of $10^{6}$ for simple optical spring damping and servoenhanced optical spring damping when using offset frequency of $f_{o s}=-f_{p} / 2$. We see that for low-frequency mechanical modes the optical input power is a few watts, but this increases to $\sim 100 \mathrm{~W}$ for modes greater than $\sim 100 \mathrm{kHz}$. If instead a shorter cavity with a somewhat narrower linewidth is chosen [case (b)], the maximum power requirement is reduced to less than $1.5 \mathrm{~W}$. A $1-\mathrm{cm}$ tranquilizer cavity with a finesse of $\mathcal{F}=30000$ (with a mirror efficiency of $\eta$ $=0.165$ ) would have $5.8 \mathrm{~kW}$ of circulating power. Given that the maximum achievable damage threshold of optical coatings is around $1 \mathrm{MW} / \mathrm{cm}^{2}$ [13], the radial spot size on the mirror would have to be greater than $0.43 \mathrm{~mm}$. A cavity formed between a flat surface on the test mass and a second concave mirror would have a $g$ factor of around 0.9997. With such a high cavity $g$ factor, the cavity-mode position on the test mass will be very sensitive to test-mass angular fluctuations. These mode position fluctuations will induce intracavity power fluctuations due to the coupling between the injection beam and the cavity mode, which consequently drives the suspended mirror via radiation pressure force and creates mirror longitudinal position noise. For Advanced LIGO, with a sensitivity of $10^{-20} \mathrm{~m} / \sqrt{\mathrm{Hz}}$ at $100 \mathrm{~Hz}$, the tranquilizer cavity would require angular control to within $\sim 10^{-8} \mathrm{rad} / \sqrt{\mathrm{Hz}}$ in order to avoid noise coupling from mirror angular fluctuations. This is comparable to the control achieved with the current LIGO. A more detailed analysis is available in Ref. [12]. In addition, the cavity needs to interact with a mechanical antinode. For the large number of mechanical modes present, this means that several cavities would be required to ensure that each cavity interacts near an appropriate antinode.

A damping cavity clearly requires the use of high-stability lasers to prevent the introduction of technical noise via radiation pressure fluctuations. For Advanced LIGO sensitivity levels, the laser intensity fluctuations inside the tranquilizer cavities have to be kept below $\sim 10^{-9} / \sqrt{\mathrm{Hz}}$ at $\sim 100 \mathrm{~Hz}$ to avoid radiation pressure force noise coupling driving the suspended test masses. A more critical noise source arises from back-action of the thermal noise from the tranquilizer cavity on the test mass. The cavity interacts only with a small area of the test mass and therefore experiences relatively large thermal noise because the bulk thermal noise scales inverse linearly with the beam spot size and the coating thermal noise scales inversely square of the beam spot size [14]. This noise modulates the tranquilizer cavity and drives the test mass via the optical spring. In the case of optimal damping we estimate that the thermal noise level in the tranquilizer cavity has to be less than $10^{-18} \mathrm{~m} / \sqrt{\mathrm{Hz}}$ at $\sim 100 \mathrm{~Hz}$ to keep the noise coupling much lower than $\sim 10^{-20} / \sqrt{\mathrm{Hz}}$.

We have demonstrated enhanced optical damping of a mechanical resonator through the introduction of a laser servo phase delay. We have shown that enhanced optical damping can allow suppression of predicted PI in advanced GW detectors at practical levels of input laser power with practically achievable mirror optical coatings; however, power density limitations require large spot sizes and therefore nearly flat-flat cavities, which require angular control within $\sim 10^{-8} \mathrm{rad}$.

This is a project of the Australian Consortium for Gravitational Astronomy, supported by the Australian Research Council and the Western Australian Government OSI. We thank the LSC Gingin Advisory Committee for assistance and support. The LIGO Observatories were constructed by Caltech and MIT with NSF funding under Grant No. PHY9210038. The LIGO Laboratory operates under cooperative agreement No. PHY-0107417. This paper has been assigned LIGO Document No. LIGO-P060061-00-Z.
[1] J. Hough and S. Rowan, J. Opt. A, Pure Appl. Opt. 7, S257 (2005).

[2] V. B. Braginsky, S. E. Strigin, and S. P. Vyatchanin, Phys. Lett. A 305, 111 (2002).

[3] C. Zhao, L. Ju, J. Degallaix, S. Gras, and D. G. Blair, Phys. Rev. Lett. 94, 121102 (2005).
[4] S. Gras, D. G. Blair, and L. Ju, www.ligo.org/pdf_public/ gras.pdf

[5] V. B. Braginsky and S. P. Vyatchanin, Phys. Lett. A 293, 228 (2002).

[6] D. G. Blair, E. N. Ivanov, M. E. Tobar, P. J. Turner, F. van Kann, I. S. Heng, Phys. Rev. Lett. 74, 1908 (1995). 
[7] O. Arcizet, P. Cohadon, T. Briant, M. Pinard, and A. Heidmann, Nature (London) 444, 71 (2006).

[8] B. Sheard, M. Gray, C. Mow-Lowry, and D. E. McClelland, Phys. Rev. A 69, 051801(R) (2004).

[9] T. Corbitt, D. Ottaway, E. Innerhofer, E. Innerhofer, J. Pelc, and N. Mavalvala, Phys. Rev. A 74, 021802(R) (2006).

[10] V. B. Braginsky, S. E. Strigin, and S. P. Vyatchanin, Phys. Lett. A 287, 331 (2001).
[11] S. W. Schediwy, S. Gras, L. Ju, and D. G. Blair, Rev. Sci. Instrum. 76, 026117 (2005).

[12] S. W. Schediwy, Ph. D. thesis, University of Western Australia, 2007.

[13] D. Reitze, www.ligo.caltech.edu/docs/G/G050360-00/ G050360-00.ppt

[14] Y. Levin, Phys. Rev. D 57, 659 (1998). 